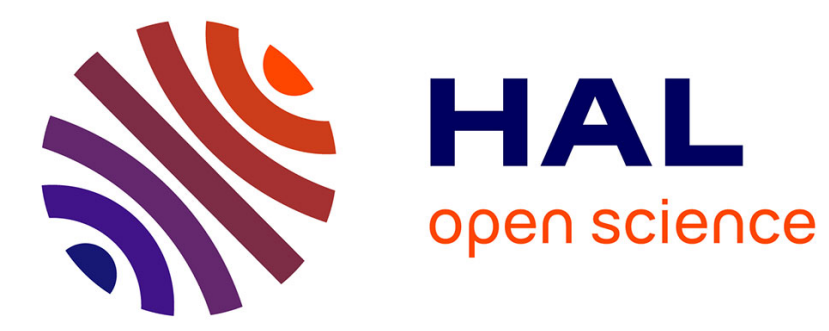

\title{
Ultrasound-based subject-specific parameters improve fascicle behaviour estimation in Hill-type muscle model
}

\author{
Pauline Gerus, Guillaume Rao, Eric Berton
}

\section{To cite this version:}

Pauline Gerus, Guillaume Rao, Eric Berton. Ultrasound-based subject-specific parameters improve fascicle behaviour estimation in Hill-type muscle model. Computer Methods in Biomechanics and Biomedical Engineering, 2015, 18 (2), pp.116 - 123. 10.1080/10255842.2013.780047 . hal-01440194

\author{
HAL Id: hal-01440194 \\ https://hal.science/hal-01440194
}

Submitted on 15 Nov 2017

HAL is a multi-disciplinary open access archive for the deposit and dissemination of scientific research documents, whether they are published or not. The documents may come from teaching and research institutions in France or abroad, or from public or private research centers.
L'archive ouverte pluridisciplinaire HAL, est destinée au dépôt et à la diffusion de documents scientifiques de niveau recherche, publiés ou non, émanant des établissements d'enseignement et de recherche français ou étrangers, des laboratoires publics ou privés. 
This article was downloaded by: [University of Nebraska, Lincoln]

On: 07 April 2015, At: 06:46

Publisher: Taylor \& Francis

Informa Ltd Registered in England and Wales Registered Number: 1072954 Registered office: Mortimer House, 37-41 Mortimer Street, London W1T 3J H, UK

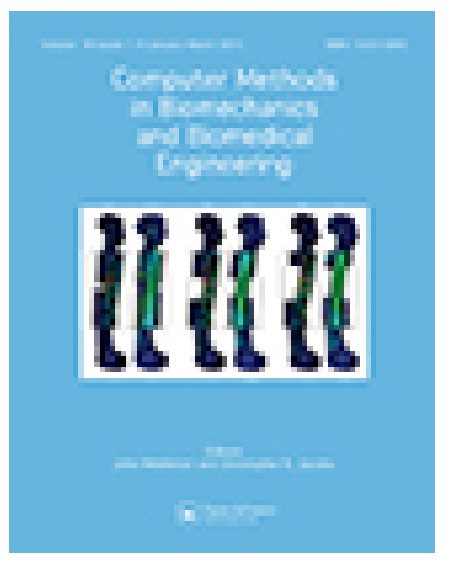

CrossMark

\section{Computer Methods in Biomechanics and Biomedical Engineering}

Publication details, including instructions for authors and subscription information: http:// www. tandfonline.com/loi/gcmb20

Ultrasound-based subject-specific parameters improve fascicle behaviour estimation in Hill-type muscle model

\author{
Pauline Gerus ${ }^{\mathrm{ab}}$, Guillaume Rao ${ }^{\mathrm{a}} \&$ Eric Berton ${ }^{\mathrm{a}}$ \\ a Institute of Movement Sciences E-J Marey, Aix-Marseille Université, Marseille, France \\ b Musculoskeletal Research Program, Griffith Health Institute, Griffith University, Gold \\ Coast Campus, Queensland 4222, Australia \\ Published online: 22 Mar 2013.
}

Click for updates

To cite this article: Pauline Gerus, Guillaume Rao \& Eric Berton (2015) Ultrasound-based subject-specific parameters improve fascicle behaviour estimation in Hill-type muscle model, Computer Methods in Biomechanics and Biomedical Engineering, 18:2, 116-123, DOI: 10.1080/ 10255842.2013.780047

To link to this article: http:// dx. doi.org/ 10.1080/ 10255842.2013.780047

\title{
PLEASE SCROLL DOWN FOR ARTICLE
}

Taylor \& Francis makes every effort to ensure the accuracy of all the information (the "Content") contained in the publications on our platform. However, Taylor \& Francis, our agents, and our licensors make no representations or warranties whatsoever as to the accuracy, completeness, or suitability for any purpose of the Content. Any opinions and views expressed in this publication are the opinions and views of the authors, and are not the views of or endorsed by Taylor \& Francis. The accuracy of the Content should not be relied upon and should be independently verified with primary sources of information. Taylor and Francis shall not be liable for any losses, actions, claims, proceedings, demands, costs, expenses, damages, and other liabilities whatsoever or howsoever caused arising directly or indirectly in connection with, in relation to or arising out of the use of the Content.

This article may be used for research, teaching, and private study purposes. Any substantial or systematic reproduction, redistribution, reselling, loan, sub-licensing, systematic supply, or distribution in any form to anyone is expressly forbidden. Terms $\&$ Conditions of access and use can be found at http:// www.tandfonline.com/page/terms-and-conditions 


\title{
Ultrasound-based subject-specific parameters improve fascicle behaviour estimation in Hill-type muscle model
}

\author{
Pauline Gerus ${ }^{\mathrm{a}, \mathrm{b} *}$, Guillaume Rao ${ }^{\mathrm{a}}$ and Eric Berton ${ }^{\mathrm{a}}$ \\ ${ }^{a}$ Institute of Movement Sciences E-J Marey, Aix-Marseille Université, Marseille, France; ${ }^{b}$ Musculoskeletal Research Program, Griffith \\ Health Institute, Griffith University, Gold Coast Campus, Queensland 4222, Australia
}

(Received 2 December 2011; final version received 22 February 2013)

\begin{abstract}
The estimation of muscle fascicle behaviour is decisive in a Hill-type model as they are related to muscle force by the forcelength-velocity relationship and the tendon force-strain relationship. This study was aimed at investigating the influence of subject-specific tendon force-strain relationship and initial fascicle geometry (IFG) on the estimation of muscle forces and fascicle behaviour during isometric contractions. Ultrasonography was used to estimate the in vivo muscle fascicle behaviour and compare the muscle fascicle length and pennation angle estimated from the Hill-type model. The calibrationprediction process of the electromyography-driven model was performed using generic or subject-specific tendon definition with or without IFG as constraint. The combination of subject-specific tendon definition and IFG led to muscle fascicle behaviour closer to ultrasound data and significant lower forces of the ankle dorsiflexor and plantarflexor muscles compared to the other conditions. Thus, subject-specific ultrasound measurements improve the accuracy of Hill-type models on muscle fascicle behaviour.
\end{abstract}

Keywords: EMG-driven model; muscle modelling; mechanical characterisation; muscle fibre

\section{Introduction}

Developing accurate models of the musculoskeletal system is challenging because of the intrinsic complexity of biological systems (Delp and Loan 2000). Hill-type muscle models were developed to account for the mechanical parameters influencing the force produced by the muscle-tendon complex where the muscle fibre is described by a contractile and parallel elastic element positioned in series with an elastic component. Whatever the neuromusculoskeletal model used (Zajac 1989; Buchanan et al. 2004; Delp et al. 2007; Seth and Pandy 2007; Hamner et al. 2010), all of them include Hill-type muscle models. This model is used widely, and thus, the question arises as to whether the used model parameters are accurate.

One important parameter to compute muscle forces from Hill-type model is the estimation of muscle fibre behaviour. Indeed, the muscle force depends on the muscle fascicle length and velocity based on a force-lengthvelocity relationship as well as on the pennation angle of the muscle fascicle relative to the tendon. The pattern of length changes is influenced by the mechanical properties of the tendon (Roberts 2002), which is generally characterised in Hill-type muscle model by a generic tendon force-strain relationship based on in vitro tests (Zajac 1989). The neuromusculoskeletal models generally represent an average adult subject, and are thus not subject-specific. In a Hill-type muscle model, the tendon force-strain relationship is generally generic whatever the subject and the task, and the fascicle length and pennation angle are computed numerically using the muscle thickness estimated from cadaveric studies. While these parameters, which have been studied separately using ultrasonography (US), are important to estimate muscle forces, no study used subject-specific data for the tendon force-strain relationship in combination with data on initial fascicle geometry (fascicle length and pennation angle (IFG)) in Hill-type models.

Previous studies were interested in including subject specificity into models such as the muscle physiological cross-sectional area (de Oliveira and Menegaldo 2010) and the optimal fibre length and pennation angle $(\mathrm{Li}$ et al. 2009) using US and showed that the use of subject-specific model parameters increase the accuracy of net joint moment or movement prediction. However, these studies were focused only on the moment prediction, which is one among many model parameters. Whereas the estimation of muscle fascicle behaviour in Hill-type muscle model is an important step to estimate muscle force, US allows the estimation of the in vivo changes in muscle fascicle length and pennation angle. The comparison of ultrasound measurement and estimation of muscle fibre behaviour appears as an additional parameter directly related to the estimation of muscle force as well as the ability of net joint moment prediction previously used to quantify the accuracy of neuromusculoskeletal models.

This study investigated the influence of the subjectspecific definition of the tendon and the constraint on

*Corresponding author. Email: pauline@gerus.fr 
initial fascicle length and pennation angle in Hill-type muscle models. The in vivo subject-specific tendon forcestrain relationship (SST) and initial fascicle length and pennation angle of the medial gastrocnemius (MG) muscle were estimated by US for each subject during static contractions. We investigated the influence of these parameters on the estimation of muscle forces and muscle fascicle behaviour using an electromyography (EMG)driven model including the Hill-type muscle model. US was used to estimate the in vivo behaviour of the muscle fascicle and compare these experimental measurements with the muscle fascicle length and pennation angle estimated from the Hill-type muscle model. We hypothesised that the combination of the subject-specific tendon definition and IFG improves the accuracy of the Hill-type model and allows obtaining muscle fascicle length and pennation angle closer to that obtained with in vivo experimental measurement.

\section{Methods}

\subsection{Experimental design}

Seven healthy males (aged $25.9 \pm 1.6$ years, body mass $72.7 \pm 9.8 \mathrm{~kg}$, height $1.77 \pm 0.05 \mathrm{~m}$ ) participated in this study. All participants gave informed written consent. Subjects were seated on the bench of a custom static ergometer with the knee fully extended and the sole of the foot perpendicular to the shank. The right foot was set in a rigid shoe fixed to a plate with both joint and static torquemeter (CS3, Meiri) axis aligned. The net ankle joint moment was sampled at $2000 \mathrm{~Hz}$. Subjects performed three 3-s maximal voluntary isometric plantarflexion contraction trials (MVIC) with verbal encouragement and on-screen joint moment feedback. Then, they performed isometric ramp-up and ramp-down contractions (from rest to $100 \%$ MVIC $)$ in $1.5 \mathrm{~s}\left(C_{1.5}\right)$ and in minimal time $\left(C_{\max }\right)$ with on-screen feedback on the expected moment. Three trials were recorded for each condition with a resting time of $2 \mathrm{~min}$. These two contraction durations were chosen to be close to the loading rate encompassed during walking $\left(C_{1.5}\right)$ or running tasks $\left(C_{\max }\right)$.

During fixed-end contractions, EMG signals were recorded at $2000 \mathrm{~Hz}$ (Trigno Wireless, Delsys, Boston, MA, USA) using surface electrodes placed over the muscles according to recommendations of the SENIAM group. Muscle activities for the lateral gastrocnemius (LG), MG, soleus (SOL) and tibialis anterior (TA) were recorded. Kinematic data of the lower limbs were recorded synchronously at $125 \mathrm{~Hz}$ (Vicon Motion System, Lake Forest, CA, USA), tracking 12 markers attached to the fifth and first metatarsal heads, the calcaneum, the lateral and medial malleoli, the fibula head, the lateral and medial condyles of the femur, the greater trochanter for both legs, the right and left anterior superior iliac spines and three markers attached to the ultrasound probe located over the muscle-tendon junction (MTJ) in order to estimate the instantaneous tendon length (Gerus et al. 2011). A first 50$\mathrm{mm}$ linear array ultrasound probe (BK-Med Pro-Focus, BK-Med, Herlev, Denmark) was positioned over the MTJ of the MG muscle. A second 40-mm linear array ultrasound probe (BK-Med Ultra-view) was fixed over the mid-belly of the MG muscle at $30 \%$ of the lower leg length (i.e. from the popliteal crease to the centre of the lateral malleolus) and aligned approximately in the same plane as the muscle line action (Oda et al. 2007). The probes were carefully attached to the leg using foam fixations and secured with elastic bandage. Images were recorded synchronously at $30 \mathrm{~Hz}$ with two image acquisition boards (PCI 1410, National Instrument, Austin, TX, USA). The synchronisation of the ultrasound video acquisition with Vicon system was performed by an analogue signal $(0-5 \mathrm{~V})$.

\subsection{Estimation of in vivo subject-specific tendon force- strain relationships and muscle fascicle geometry}

Ultrasound images were used to track the MTJ defined as the intersection between the MG muscle fascicles and the MG tendon. Raw images were processed by adjusting the contrast and then binarised using a grey threshold value. The MTJ displacements were tracked using the method presented by Korstanje et al. (2010) with manual adjustment when necessary. With this method, all possible pixel displacements within the search region were evaluated using normalised cross-correlation, while the higher value was used to determine the MTJ position on the next frame. On each frame, the tendon length was computed as the distance between the marker placed on calcaneum and the MTJ position in the global coordinate system using the probe reference frame (more details in Gerus et al. 2011). The tendon strain of the MG muscle $\left(\varepsilon^{\mathrm{T}}\right)$ was defined as

$$
\varepsilon^{\mathrm{T}}=\frac{\Delta l}{l_{0}}
$$

where $l_{O}$ is the initial tendon length computed in the rest position and is the elongation of the tendon.

Normalised MG force was estimated from the net ankle joint moment by assuming that the moment arm of MG tendon (Maganaris et al. 2004) and the relative moment contribution of MG muscle to the net joint moment (Kubo et al. 2004) remained constant during ramp-up contraction. Finally, a second-order polynomial model was used to fit each tendon force-strain relationship for each subject and condition (i.e. $C_{1.5}$ and $\left.C_{\max }\right)$.

The muscle fascicle length and pennation angle of the MG muscle were computed from ultrasound data. The muscle fascicle length was defined as the distance between 


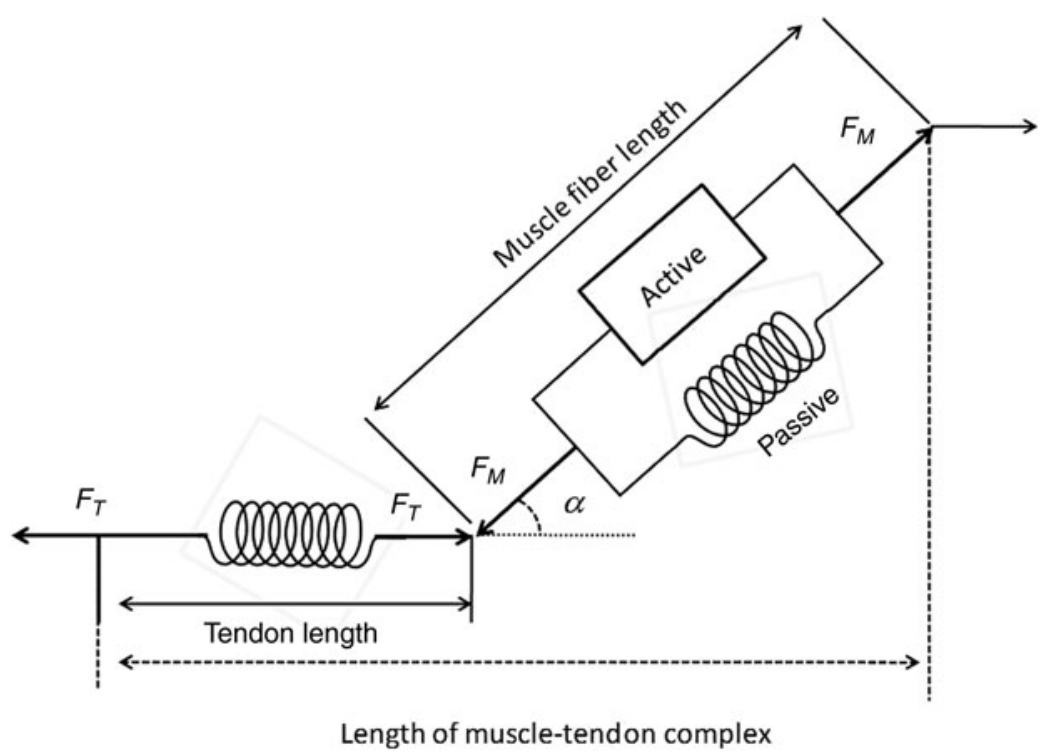

Figure 1. Representation of muscle-tendon complex in a Hill-type muscle model. The muscle fibre is in series with the tendon, which represents the series elastic component. The force produced by the muscle fibre is a function of an active and passive component and the pennation angle $\alpha$, while the tendon force is a function of the tendon length. The active component includes the active force-length relationship and the force-velocity relationship, and the passive component is represented by the passive force-length relationship.

the intersection of the fascicle with the superficial and deeper aponeurosis. The entire muscle fascicle could not be seen on the US image; thus, the missing part of fascicle was estimated by linear extrapolation of both aponeurosis and fascicle (Finni and Komi 2002). The pennation angle was defined as the angle between the deeper aponeurosis and the muscle fascicle. The fascicle length and pennation angle from three fascicles were determined manually on the first frame. Then, the proximal and distal ends of each fascicle visible on the frame were tracked automatically for each following frame by the same method used to track the MTJ. Manual adjustments were made if necessary. The fascicle length and pennation angle of the three fascicles were averaged on each frame to obtain a representative fibre behaviour during the contraction. The initial fascicle length and pennation angle of the MG muscle were determined from the beginning of net ankle joint moment production in order to obtain a set of IFG for each subject and condition.

\subsection{Estimation of muscle forces}

The model used in the present study has already been extensively described (Buchanan et al. 2004), and only a brief description is given here. The EMG-driven model used EMG, kinematic, anatomical and net joint moment data to estimate muscle forces. The model consisted of five parts: (1) an anatomical model to estimate muscle-tendon lengths and moment arms, (2) an EMG-to-activation model to represent muscle activation dynamics, (3) a
Hill-type model (Figure 1) to characterise muscle-tendon contraction dynamics and estimate the forces in the muscle-tendon complex, (4) a calibration process to tune model parameters based on net ankle joint moment recorded by the static torquemeter and (5) a prediction process to predict joint moment by using adjusted model parameters.

Using OpenSim (Simtk, Stanford, CA, USA), the constant muscle-tendon lengths and flexion-extension moment arms of the four muscles were estimated for each subject from a scaled model with the knee fully extended and the ankle angle at $0^{\circ}$ (more information in Delp et al. 2007). The raw EMG were band-pass filtered (10$500 \mathrm{~Hz}$ ), full-wave rectified and filtered using a zero-lag Butterworth low-pass filtre (4th order, 6-Hz cut-off frequency). The processed EMG, muscle-tendon lengths, flexion-extension moment arms of LG, MG, SOL and TA and the net ankle joint moment of each trial were used as input in the EMG-driven model.

During the calibration process, the model parameters were adjusted using numerical optimisation under constraints by minimising the difference between the net ankle joint moment computed by the model and the net ankle joint moment recorded by the static torquemeter. Two tendon definitions of the Hill-type model (Figure 1) were used during the calibration process:

- The in vivo SSTs obtained previously on the MG muscle for each contraction using US. 
Table 1. Summary of different calibration conditions. 'Subject-specific' corresponds to the tendon force-strain relationships obtained by US for each subject on the MG muscle and used for all muscles. 'Generic' corresponds to the generic tendon force-strain relationship described by Zajac.

\begin{tabular}{llr}
\hline Tendon definition & IFG for the MG muscle (fascicle length and pennation angle) & Name of calibration \\
\hline Generic & No constraint & GT \\
Generic & Constrained & GT-IFG \\
Subject-specific & No constraint & SST \\
Subject-specific & Constrained & SST-IFG \\
\hline
\end{tabular}

- The generic curve defined by Zajac (1989) given by

$$
\left\{\begin{array}{cc}
\bar{F}^{\mathrm{T}}=0 & \varepsilon^{\mathrm{T}} \leq 0 \\
\bar{F}^{\mathrm{T}}=1480.3 \varepsilon^{2 \mathrm{~T}} & 0<\varepsilon^{\mathrm{T}}<0.0127, \\
\bar{F}^{\mathrm{T}}=37.5 \varepsilon^{\mathrm{T}}-0.2375 & \varepsilon^{\mathrm{T}} \geq 0.0127
\end{array}\right.
$$

with $\bar{F}_{\mathrm{T}}$ and $\varepsilon_{\mathrm{T}}$ being the tendon force and tendon strain, respectively.

The IFG (for the first data point) of the MG muscle was either numerically computed during the optimisation process or constrained from the US data. The initial fascicle length was used only for the MG muscle at the beginning of each task. The estimation of fibre length was numerically computed for the remaining data points and other muscles. The EMG-driven model calibration process was performed simultaneously on one trial of each fixedend contraction condition $\left(C_{1.5 \mathrm{~s}}\right.$ and $\left.C_{\max }\right)$ with (1) the generic Zajac curve (GT), (2) Zajac combined with initial fascicle length and pennation angle of the MG muscle (GT-IFG), (3) in vivo SSTs and (4) in vivo SSTs combined with initial fascicle length and pennation angle of the $\mathrm{MG}$ muscle (SST-IFG). An overview of the different calibration conditions is given in Table 1 .

Once the calibration process was achieved, the adjusted model parameters were used to predict the ankle moment using the EMG and kinematic data from other trials. During the prediction process, the two tendon definitions were used. The data obtained from this section were further named prediction.

Table 2. Summary of parameters computed from ultrasound data according to condition (mean $\pm \mathrm{SD}$ ).

\begin{tabular}{lcc}
\hline & $C_{\max }$ & $C_{1.5}$ \\
\hline Resting tendon length (mm) & $208 \pm 32$ & $207 \pm 32$ \\
Tendon elongation (mm) & $14.6 \pm 2.5^{\mathrm{a}}$ & $17.0 \pm 3.6^{\mathrm{a}}$ \\
Tendon strain (\%) & $7.2 \pm 1.9^{\mathrm{a}}$ & $8.4 \pm 2.5^{\mathrm{a}}$ \\
Initial fascicle length & $59.4 \pm 3.3$ & $59.6 \pm 3.9$ \\
Amplitude of fascicle length (mm) & $20.9 \pm 4.8$ & $22.6 \pm 3.4$ \\
Initial pennation angle & $20.2 \pm 2.6$ & $19.9 \pm 2.0$ \\
Amplitude of pennation angle & $9.5 \pm 2.2$ & $10.9 \pm 2.7$ \\
Muscle thickness & $20.3 \pm 2.5$ & $20.3 \pm 2.1$ \\
\hline
\end{tabular}

${ }^{\mathrm{a}}$ Reveal significant difference between condition $C_{\max }$ and $C_{1.5}$.

\subsection{Data analysis}

After the calibration and prediction processes, the net joint moment estimated by the model was compared with the net joint moment recorded by the static torquemeter. A coefficient of determination (R2) was used to indicate the closeness of fit in conjunction with the slope and intercept to indicate the linear relationship between estimated and measured flexion-extension (FE) moment, and the mean residual error to indicate the magnitude of the error.

For each prediction, the outputs of the model were studied through the following data: the maximal muscle forces, the amplitude (maximum value-minimum value) of muscle forces, fascicle lengths, pennation angles and maximal tendon strains. The fascicle length and pennation angle determined at each time by ultrasound were used as reference and compared with the output of the model using root-mean-square error $\left(\mathrm{RMS}_{\text {error }}\right)$ computed as

$$
\mathrm{RMS}_{\text {error }}=\left(\frac{1}{n} \cdot \sum_{i=1}^{n}\left(\mathrm{US}\left(t_{i}\right)-\operatorname{Mod}\left(t_{i}\right)\right)^{2}\right)^{1 / 2},
$$

where $n$ represents the number of samples, US and Mod represent the US data and the model output (i.e. fascicle length or pennation angle), respectively.

A one-factor (calibrations) analysis of variance (ANOVA) with repeated measures was conducted on the amplitude and maximal value of muscle force, the amplitude of fascicle length, pennation angle and tendon strain for the LG, MG, SOL and TA. A one-factor (conditions and US) ANOVA with repeated measures was conducted on the amplitude of muscle fascicle length and pennation angle obtained from US and model. Finally, a one-factor (conditions) ANOVA with repeated measures was conducted on the $\mathrm{RMS}_{\text {error }}$ of fascicle length and pennation angle. A significance level of 0.05 was used for all comparisons, and Newman-Keuls post hoc testing was used whenever necessary.

\section{Results}

The maximal strain of the MG tendon obtained by in vivo measurement ranged from $4.9 \%$ to $12.7 \%$, and the amplitude of MG fascicle length and MG pennation angle ranged from 12.4 to $26.6 \mathrm{~mm}$ and from 6.4 to $14.4^{\circ}$, 


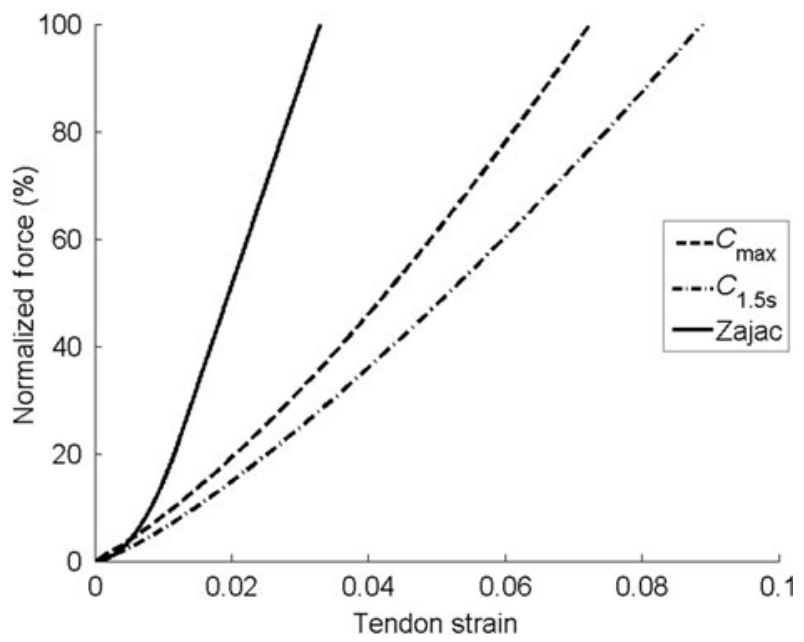

Figure 2. Group-averaged subject-specific tendon force-strain relationships of the MG tendon estimated by ultrasonography. $C_{\max }$ and $C_{1.5}$ represent the different isometric contractions, where the ramp-up time from $0 \%$ to $100 \%$ of MVC was minimal and in $1.5 \mathrm{~s}$, respectively. The generic tendon force-strain relationship described by Zajac and used in the calibration and prediction processes is also presented for comparison.

respectively (Table 2). The in vivo subject-specific mechanical definition of tendon revealed a more compliant tendon than the generic definition described by Zajac (Figure 2).

The averaged mean residual error and $\mathrm{R} 2$ for the calibration process was $0.95( \pm 0.03)$ and $10.8( \pm 2.0$ N.m $)$, respectively. The averaged mean residual error and $\mathrm{R} 2$ for the prediction process was $0.92( \pm 0.07)$ and 14.0 $( \pm 5.9 \mathrm{~N} . \mathrm{m})$, respectively, without significant difference between the different conditions. After model prediction, the amplitude of the tendon displacement, the muscle fascicle length and the pennation angle for $\mathrm{LG}, \mathrm{MG}$ and SOL were significantly higher for the SST-IFG and SST conditions compared with GT and GT-IFG (Table 3). The maximal value of the MG muscle force was significantly higher for GT, GT-IFG and SST compared with SST-IFG (Table 4 and Figure 4). For the LG and TA muscles, the use of in vivo SST and SST-IFG influenced the estimation of muscle forces by decreasing the maximal value.
The amplitude of fascicle length and pennation angle for SST-IFG during fixed-end contractions showed similar behaviour than US data as opposed to other conditions (Table 3). Similarly, a lower RMS error for fascicle length and pennation angle were found in the SST-IFG condition (Figures 3 and 4). The use of GT definition leads to a lower amplitude of muscle fascicle length and pennation angle compared with US data, whatever the muscle fascicle geometry used in the EMG-driven model (Table 3).

\section{Discussion}

The present study was aimed at identifying the subjectspecific properties of the muscle-tendon complex that improve the accuracy of fascicle behaviour estimated by the Hill-type muscle model. In this study, the influence of the tendon force-strain relationship and IFG of the MG muscle in the EMG-driven model has been investigated on model outputs (i.e. muscle force and fascicle behaviour). The estimated in vivo SSTs were significantly more compliant than the generic tendon definition described by Zajac, whatever the duration of contraction. The estimation of the LG and TA muscle forces for the in vivo SST-IFG condition was lower compared with the calibration with the generic tendon definition described by Zajac. The MG muscle force was lower for prediction with SST-IFG compared with the other conditions and had the closest trajectory of muscle fibre length and pennation angle with the ultrasound measurement.

The use of the in vivo subject-specific tendon definition leads to significantly higher tendon strain, muscle fascicle length and pennation angle for LG, MG and SOL compared with the use of the generic tendon definition described by Zajac. The explanation of these differences lies in the more compliant tendon definition for the in vivo SST compared with the generic tendon definition described by Zajac as the maximal strain of MG tendon observed during fixed-end contraction ranged from $4.9 \%$ to $12.7 \%$ for all conditions. Our results agreed with previous results (Griffiths 1991; Maganaris and Paul 2000; Monti et al. 2003; Arampatzis et al. 2005). The in vivo values of muscle architecture parameters obtained in this study lay among the previously reported values. Indeed,

Table 3. Summary of muscle-tendon parameters for the MG muscle depending on model conditions. Lf represents the fascicle length and $\theta$ represents the pennation angle.

\begin{tabular}{lccrrr}
\hline & US & SST & SST-IFG & GT & GT-IFG \\
\hline RMS $_{\text {error }}$ Lf & & $7.5 \pm 4.2^{*}$ & $4.3 \pm 1.3^{*}$ & $13.1 \pm 5.9^{*}$ & $10.7 \pm 4.7^{*}$ \\
RMS $_{\text {error }} \theta$ & $8.2 \pm 2.3^{*}$ & $3.2 \pm 1.8^{*}$ & $7.9 \pm 4.0^{*}$ & $4.2 \pm 1.6^{*}$ \\
Amplitude of Lf $(\mathrm{mm})$ & $19.4 \pm 3.8$ & $22.5 \pm 5.1^{* *}$ & $18.6 \pm 3.89$ & $7.0 \pm 1.5^{* *}$ & $7.2 \pm 1.6^{* *}$ \\
Amplitude of $\theta\left({ }^{\circ}\right)$ & $10.2 \pm 2.5$ & $7.0 \pm 3.0^{* *}$ & $10.4 \pm 4.5$ & $2.7 \pm 1.4^{* *}$ & $2.6 \pm 0.6^{* *}$ \\
Muscle thickness & $20.3 \pm 2.2$ & $13.7 \pm 1.0^{* *}$ & $20.3 \pm 2.2$ (US) & $13.8 \pm 1.1^{* *}$ & $20.3 \pm 2.2$ (US) \\
\hline
\end{tabular}

* Indicate significant difference between SST-IFG and the other conditions.

${ }^{* * *}$ Indicate significant difference between US data and conditions followed by SST, GT and GT-IFG. 
Table 4. Summary of muscle forces depending on model conditions.

\begin{tabular}{lcrrr}
\hline Force $\max (\mathrm{N})$ & SST & SST-IFG & GT & GT-IFG \\
\hline LG & $427.1 \pm 123.2$ & $429.0 \pm 115.4^{*}$ & $474.5 \pm 140.5^{*}$ & $468.8 \pm 145.1^{*}$ \\
MG & $968.9 \pm 204.5^{*}$ & $840.8 \pm 209.8^{*}$ & $964.7 \pm 224.7^{*}$ & $1041.4 \pm 253.7^{*}$ \\
SOL & $1779.4 \pm 497.8$ & $1788.2 \pm 480.5$ & $1933.5 \pm 572.9$ & $1818.8 \pm 552.9$ \\
TA & $122.8 \pm 77.1$ & $113.3 \pm 86.2^{*}$ & $174.1 \pm 70.5^{*}$ & $171.8 \pm 72.2^{*}$ \\
\hline
\end{tabular}

*Indicate significant difference between SST-IFG and the other conditions.

previous studies have reported that the human fascicle length and the pennation angle of the MG muscle vary between 50.8 and $60.0 \mathrm{~mm}$ and between 17.3 and $24.1^{\circ}$ in rest position, and between 30.0 and 32.9 and between 34.6 and $40.8^{\circ}$ for $100 \%$ of $\mathrm{MVC}$, respectively and that the muscle thickness is about $22.8 \mathrm{~mm}$ Narici et al. 1996; Kawakami et al. 1998; Kubo et al. 2000; Kurihara et al. 2005; Arampatzis et al. 2007; Manal et al. 2008). The use of initial fascicle length and pennation angle combined with in vivo SST contributes to significantly increase the amplitude of the pennation angle. The pennation angle is related to the muscle force projection along the tendon force axis, and thus, an increase in pennation angle leads to a decrease in muscle force projection.

Our results indicate that in an EMG-driven model and more generally, in a Hill-type muscle model both the tendon definition and IFG influence the estimation of muscle forces. Indeed, the maximal value of the $\mathrm{MG}$ muscle force for the condition including in vivo SSTs combined with initial fascicle length and pennation angle was significantly lower than that in the GT, GT-IFG and SST conditions. The different behaviour observed on muscle fibre length and pennation angle after model prediction may explain our finding on muscle force estimation. Interestingly, adding constraint on the fascicle geometry for the MG muscle also modifies the other muscle forces. For the LG and TA muscles, the use of in vivo SST decreases the estimated muscle forces.

de Oliveira and Menegaldo (2010) and Li et al. (2009) have found that the use of subject-specific parameters increases the accuracy of net joint moment or movement prediction. In our study, there is no difference between the different conditions in terms of net joint moment prediction. Another way was proposed to quantify the effect of subject-specific parameters into the Hill-type muscle model. The US was used to quantify change in fascicle length and pennation angle during fixed-end contraction in order to compare these experimental measurements with the model outputs. The best match between the output model parameters and US data,
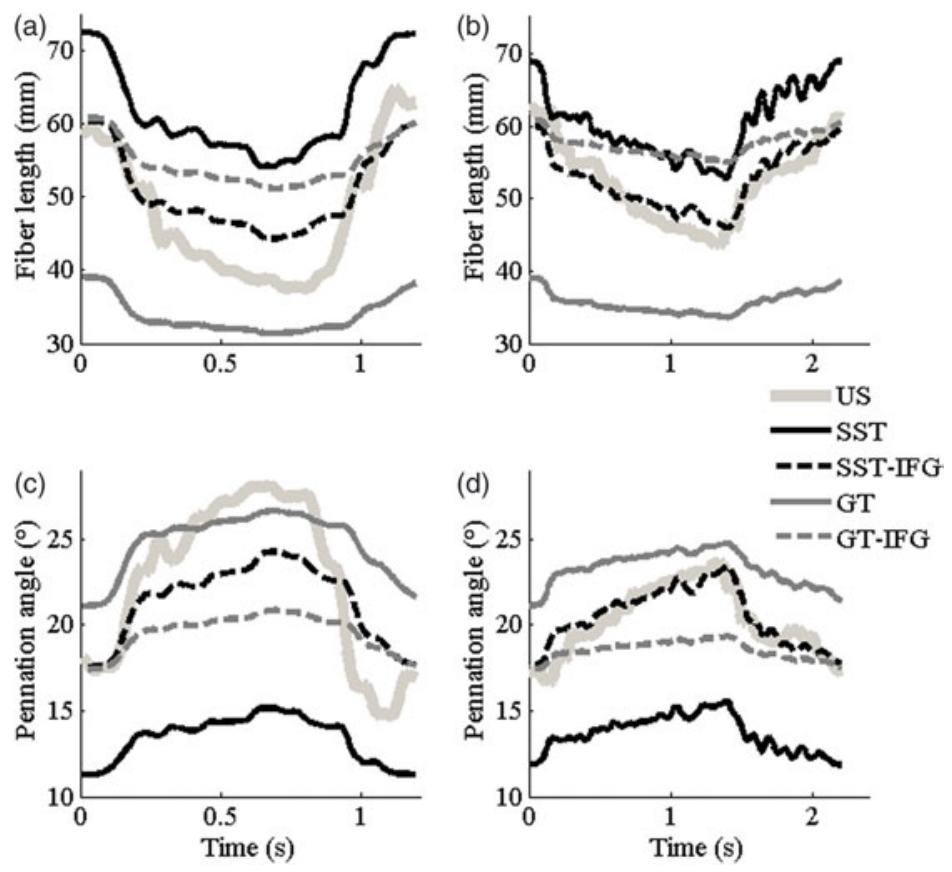

Figure 3. Muscle fascicle length and pennation angle for one subject obtained from ultrasound data and from EMG-driven model during prediction process for $C_{\max }$ and $C_{1.5}$. The initial fibre length and initial pennation angle was similar to ultrasound measurements for conditions with initial fascicle geometry. Note the closeness of the prediction SST-IFG and ultrasound measurement. 


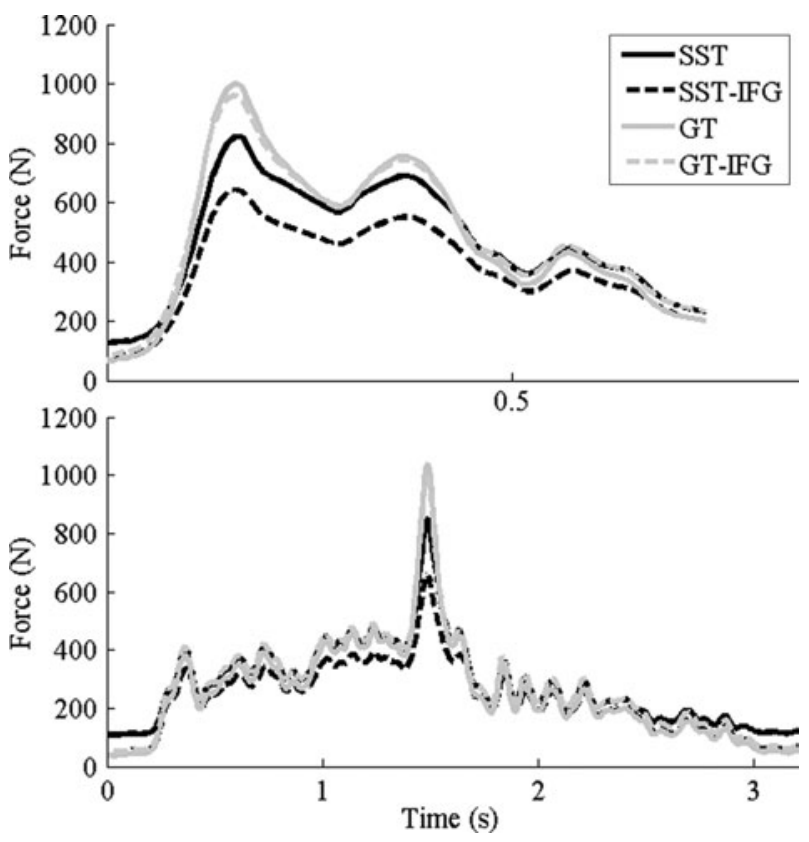

Figure 4. MG muscle force for one subject on $C_{\max }$ (top) and $C_{1.5}$ (bottom) during the prediction process. Note the lower muscle force for calibrating SST-IFG compared with the other conditions.

corresponding to lower $\mathrm{RMS}_{\text {error }}$, was found for the in vivo SST-IFG condition. These results suggest that the behaviour of muscle fascicle using subject-specific muscle-tendon properties (SSTs, initial fascicle length and pennation angle of $\mathrm{MG}$ ) is more accurate than with generic data. However, this method failed to validate the model in terms of muscle force estimation, but the similarity between muscle fascicle behaviour (i.e. the fascicle length and pennation angle) estimated by US and computed by the in vivo SST-IFG condition allows us to be confident in model outputs directly used to estimate muscle forces through the use of the force-lengthvelocity relationship.

In this study, we were focused on the SST and initial fascicle length and pennation angle. Li et al. (2009) have shown that the use of subject-specific optimal fibre length and pennation angle allows the improved prediction of elbow movement for healthy people and stroke survivors. US has been used to estimate the subject-specific moment arm (Manal et al. 2010). Further studies are necessary to investigate the influence of additional muscle-tendon parameters in EMG-driven models, such as moment arm, and combine in vivo tendon force-strain relationship, initial and optimal fibre length and pennation angle.

To conclude, ultrasound measurement appears to be useful to include subject-specific properties of the muscle-tendon complex in an EMG-driven model. The integration of both in vivo SST and IFG obtained by
US into a Hill-type muscle model leads to a closer behaviour of fibre length and pennation angle compared with US measurement and decreases the maximal force of the dorsiflexor and plantarflexor muscles. By comparing the outputs of a Hill-type muscle model (fascicle length and pennation angle) with the US data, this study proposed a method to determine the accuracy of model outputs directly used to estimate muscle forces.

\section{References}

Arampatzis A, Mademlia L, De Montea G, Walsh M. 2007. Changes in fascicle length from rest to maximal voluntary contraction affect the assessment of voluntary activation. J Biomech. 40(14):3193-3200.

Arampatzis A, Stafilidis S, DeMonte G, Karamanidis K, MoreyKlapsing G, Bruggemann GP. 2005. Strain and elongation of the human gastrocnemius tendon and aponeurosis during maximal plantarflexion effort. J Biomech. 38(4):833-841.

Buchanan TS, Lloyd DG, Manal K, Besier TF. 2004. Neuromusculoskeletal modeling: estimation of muscle forces and joint moments and movements from measurements of neural command. J Appl Biomech. 20(4):367-395.

de Oliveira LF, Menegaldo LL. 2010. Individual-specific muscle maximum force estimation using ultrasound for ankle joint torque prediction using an emg-driven hill-type model. J Biomech. 43(14):2816-2821.

Delp SL, Anderson FC, Arnold AS, Loan P, Habib A, John CT, Guendelman E, Thelen DG. 2007. Opensim: open-source software to create and analyze dynamic simulations of movement. IEEE Trans Biomed Eng. 54(11):1940-1950.

Delp SL, Loan P. 2000. A computational framework for simulating and analysing human and animal movement. Comput Sci Eng. 2(5):46-55.

Finni T, Komi PV. 2002. Two method for estimating tendinous tissue elongation during human movement. J Appl Biomech. 18(2):180-188.

Gerus P, Rao G, Berton E. 2011. A method to characterize in vivo tendon force-strain relationship by combining ultrasonography, motion capture and loading rates. J Biomech. 44(12):2333-2336.

Griffiths RI. 1991. Shortening of muscle fibres during stretch of the active cat medial gastrocnemius muscle: the role of tendon compliance. J Physiol. 436(1):219-236.

Hamner SR, Seth A, Delp SL. 2010. Muscle contributions to propulsion and support during running. J Biomech. 43(14):2709-2716.

Kawakami Y, Ichinose Y, Fukunaga T. 1998. Architectural and functional features of human triceps surae muscles during contraction. J Appl Physiol. 85(2):398-404.

Korstanje J-WH, Selles RW, Stam HJ, Hovius SER, Boshc JG. 2010. Development and validation of ultrasound speckle tracking to quantify tendon displacement. J Biomech. 43(7): $1373-1379$.

Kubo K, Akima H, Ushiyama J, Tabata I, Fukuoka H, Kanehisa H, Fukunaga T. 2004. Effects of 20 days of bed rest on the viscoelastic properties of tendon structures in lower limb muscles. Br J Sports Med. 38(3):324-330.

Kubo K, Kanehisa H, Kawakami Y, Fukunaga T. 2000. Elasticity of tendon structures of the lower limbs in sprinters. Acta Physiol Scand. 168(2):327-335.

Kurihara T, Oda T, Chino K, Kanehisa H, Fukunaga T, Kawakami Y. 2005. Use of three-dimensional ultrasono- 
graphy for the analysis of the fascicle length of human gastrocnemius muscle during contractions. Int J Sport Health Sci.3(Special_Issue_2005):226-234.

Li L, Tong KY, Hu XL, Koo TKK. 2009. Incorporating ultrasound-measured musculotendon parameters to subjectspecific EMG-driven model to simulate voluntary elbow flexion for persons after stroke. Clin Biomech. 24(1):101-109.

Maganaris CN, Narici MV, Reeves ND. 2004. In-vivo human tendon mechanical properties: effect of resistance training in old age. J Musculoskelet Neuronal Interact. 4(2):204-208.

Maganaris CN, Paul JP. 2000. Load-elongation characteristics of in-vivo human tendon and aponeurosis. $J$ Exp Biol. 203(4):751-756.

Manal K, Roberts DP, Buchanan TS. 2008. Can pennation angles be predicted from emgs for the primary ankle plantar and dorsiflexors during isometric contractions? J Biomech. 41(11):2492-2497.

Manal K, Cowder JD, Buchanan TS. 2010. A hybrid method for computing achilles tendon moment arm using ultrasound and motion analysis. J Appl Biomech. 26(2):224-228.
Monti RJ, Roy RR, Zhong H, Edgerton VR. 2003. Mechanical properties of rat soleus aponeurosis and tendon during variable recruitment in situ. J Exp Biol. 206(Pt19):3437-3445.

Narici MV, Binzoni T, Hiltbrand E, Fasel J, Terrier F, Cerretelli P. 1996. In-vivo human gastrocnemius architecture with changing joint angle at rest and during graded isometric contraction. J Physiol. 496(Pt 1):287-297.

Oda T, Kanehisa H, Chino K, Kurihara T, Nagayoshi T, Fukunaga T, Kawakami Y. 2007. In-vivo behavior of muscle fascicles and tendinous tissues of human gastrocnemius and soleus muscles during twitch contraction. J Electromyogr Kinesiol. 17(5):587-595.

Roberts TJ. 2002. The integrated function of muscles and tendons during locomotion. Comp Biochem Physiol A. 133(4):1087-1099.

Seth A, Pandy MG. 2007. A neuromusculoskeletal tracking method for estimating individual muscle forces in human movement. J Biomech. 40(2):356-366.

Zajac FE. 1989. Muscle and tendon: properties, models, scaling, and application to biomechanics and motor control. Crit Rev Biomed Eng. 17(4):359-411. 